\title{
Plastisch-chirurgisches Management bei Weichgewebesarkomen an der unteren Extremität
}

\author{
Plastic Surgical Management in Lower Extremity Sarcoma Reconstruction
}

Autoren

Institute

\section{T. Schloßhauer', I. M. Mehling', W. Moll' , G. Germann², M. Sauerbier}

${ }^{1}$ Abteilung für Plastische, Hand- und Rekonstruktive Chirurgie, Berufsgenossenschaftliche Unfallklinik Frankfurt am Main, Frankfurt am Main

${ }^{2}$ ETHIANUM - Klinik für Plastische, Ästhetische und Handchirurgie, Heidelberg
Schlüsselwörter

- freie Lappenplastiken

- Unterschenkelrekonstruktion

- untere Extremität

- Weichgewebesarkomen

Key words

- free flaps

- lower extremity reconstruction

- lower extremity

soft-tissue sarcoma

eingereicht 25.2.2015 akzeptiert 11.3.2015

Bibliografie

DOI http://dx.doi.org/ 10.1055/s-0035-1548798 Handchir Mikrochir Plast Chir 2015; 47: 100-110

(c) Georg Thieme Verlag KG Stuttgart · New York ISSN 0722-1819

Korrespondenzadresse

Prof. Dr. med. Dr. med. habil. Michael Sauerbier

Abteilung für Plastische, Handund Rekonstruktive Chirurgie Berufsgenossenschaftliche Unfallklinik Frankfurt am Main Friedberger Landstraße 430 60389 Frankfurt am Main michael.sauerbier@bgufrankfurt.de

\section{Zusammenfassung}

Tumoren im Bereich der unteren Extremität, die eine kritische Größe von über $5 \mathrm{~cm}$ Durchmesser überschreiten, sollten grundsätzlich hinsichtlich einer möglichen Malignität bzw. der Existenz eines Weichgewebesarkoms abgeklärt werden. Handelt es sich um ein Weichgewebesarkom, ist die radikale onkologische Resektion mit ausreichend weitem Sicherheitsabstand der wichtigste Eckpfeiler einer kurativen Behandlung. Keine neoadjuvante oder adjuvante Behandlung (Strahlentherapie oder Chemotherapie) kann diesen Therapieansatz ersetzen. Die modernen Verfahren der Tumorresektion sowie der plastischrekonstruktiven Chirurgie erlauben, in mehr als 95\% der Fälle eine radikale Tumorexzision durchzuführen und auch große Defekte zu verschließen sowie die Funktion der Extremität durch Muskel-, Sehnen- und Knochenverpflanzungen sowie Nerven- und Gefäßtransplantationen weitgehend zu erhalten. Plastische Rekonstruktionen nach radikaler Tumorresektion sind vielfach anspruchsvoll und komplex und erfordern in der multimodalen Therapie von Weichgewebesarkomen intensive interdisziplinäre Kooperationen. Sie beinhalten das gesamte Spektrum plastisch-chirurgischer Optionen, sollten in spezialisierten Zentren durchgeführt werden und spezifisch an den Patienten und sein Fallprofil angepasst werden. In dieser Übersichtsarbeit werden verschiedene Möglichkeiten der funktionellen Wiederherstellung nach radikal-onkologischer Entfernung von Weichgewebesarkomen beleuchtet und dargestellt.

\section{Einleitung}

\section{$\nabla$}

Weichgewebesarkome stellen eine sehr heterogene Gruppe maligner Erkrankungen mit einer geringen Inzidenz dar. Sie können in jedem Kör-

\section{Abstract}

Tumors in the lower extremity with a critical size of over $5 \mathrm{~cm}$ diameter should principally be tested for being malignant soft-tissue sarcomas. If a soft-tissue sarcoma is present, radical oncological resection with sufficiently wide surgical margins is the most important cornerstone of curative therapy. No neoadjuvant or adjuvant treatment (radiotherapy or chemotherapy) can replace this treatment approach. Modern techniques of tumor resection as well as plastic-reconstructive surgery permit one to perform radical tumor excision in more than $95 \%$ of cases and to close large defects and to largely preserve function of the extremity by transplantation of muscles, tendons and bones as well as transplantations of nerves and blood vessels. The plastic reconstructions after radical tumor resection are often demanding and complex and require intensive interdisciplinary cooperation. This consists of the full spectrum of plastic surgical options, which should be performed in specialized centers and be specifically adapted to the patient and case profile. In this review different options for functional reconstruction after radical oncological removal of soft-tissue sarcomas are presented. 
kome am häufigsten an der unteren Extremität lokalisiert, während die oberen Extremitäten weniger häufig betroffen sind ( 74 vs. $26 \%$; [2]).

Wichtigstes Ziel bei der Behandlung von Weichgewebesarkomen ist die kurative Intention verbunden mit radikaler onkologischer Tumorresektion (R0-Resektion) und ausreichendem Sicherheitsabstand. Mithilfe der modernen plastischen Chirurgie gelingt es heute, Extremitäten in über 95\% der Fälle durch funktionelle Wiederherstellungsverfahren einschließlich verschiedenster Weichteildeckungsmaßnahmen zu erhalten [3-5]. Die funktionelle Rekonstruktion nach Entfernung von Weichgewebesarkomen im Bereich der unteren Extremität unterscheidet sich in 5 Gesichtspunkten von derjenigen der oberen Extremität $[3,6]$ :

1. Stabilität und Belastbarkeit werden im Bereich der unteren Extremität gewöhnlich höher bewertet als die funktionelle Mobilität und der Bewegungsumfang.

2. Das postoperative Erscheinungsbild ist weniger relevant. Rekonstruierte Beine mit Narben und potenziell voluminösen Lappen können leicht durch die Kleidung verdeckt werden und spielen im Sozialkontakt eine geringere Rolle als im Bereich der oberen Extremität.

3. Die Belastung der unteren Extremität ist höher; der arteriosklerotische Gefäßschaden sowie der orthostatische Venendruck sind relevanter als es bei der oberen Extremität der Fall ist. Beides kann bei der freien Gewebeübertragung eine wichtige Rolle spielen.

4. Die Nervenregeneration verläuft in jedem Alter im Bereich der unteren Extremität weniger erfolgreich.

5. Die Wundheilung erfolgt langsamer und das Risiko einer Komplikation durch eine Infektion ist höher.

Entscheidend für den Behandlungserfolg ist die interdisziplinäre Zusammenarbeit des tumorresezierenden Chirurgen mit den anderen an der multimodalen Therapie beteiligten Fachdisziplinen [7]. Zu diesen gehören bspw. im Bereich der unteren Extremität Orthopäden, Pathologen, Strahlentherapeuten, Onkologen, Physio- und Ergotherapeuten sowie Orthopädietechniker.

Ziel dieses Artikels ist es, einen Überblick über die Optionen der plastisch-chirurgischen Rekonstruktionsverfahren bei der Behandlung von Weichgewebesarkomen an der unteren Extremität zu geben und Überlebensraten, die Prognose und das Outcome der Patienten zu bewerten.

\section{Epidemiologie und Ätiologie}

Weichgewebetumoren sind eine sehr heterogene Gruppe von mehr als 100 verschiedenen Tumorentitäten, die nach ihrer wichtigsten adulten Gewebekomponente eingeteilt werden. Die maligne Untergruppe der Weichgewebetumoren heißt Sarkome (abgeleitet aus dem griechischen Wort $\sigma \alpha \rho \zeta$, sarx oder Fleisch), welche nicht nur lokal invasiv wachsen können oder sogar destruktives Wachstum zeigen, sondern auch ein variables Rezidiv-Risiko und Potenzial zur Metastasenbildung haben.

Mit einer Inzidenz von ca. 2-4 Neuerkrankungen pro 100000 Einwohner pro Jahr [8] in Deutschland machen Weichgewebesarkome nur einen Anteil von ca. 1\% aller malignen Neoplasien im Erwachsenenalter und von etwa 7\% der Krebserkrankungen im Kindesalter aus. Es handelt sich also um eine seltene Erkrankung, zu der Angaben in offiziellen Krebsstatistiken fehlen. In den USA liegt die Inzidenz von Weichgewebesarkomen ver-
Tab. 1 Prädisponierende Risikofaktoren für Weichgewebesarkome [3].

\begin{tabular}{|c|c|}
\hline \multicolumn{2}{|l|}{ Risikofaktoren } \\
\hline genetische & $\begin{array}{l}\text { Neurofibromatose NF-1 } \\
\text { (Von-Recklinghausen Syndrom) } \\
\text { Retinoblastom } \\
\text { Gardner Syndrom } \\
\text { Werner Syndrom } \\
\text { Bloom Syndrom } \\
\text { Fumarat-Hydratase Leiomyosarkom Syndrom } \\
\text { Diamond-Blackfan-Anämie }\end{array}$ \\
\hline mechanische & $\begin{array}{l}\text { Li-Fraumeni Syndrom } \\
\text { nach einer Geburt }\end{array}$ \\
\hline chemische & $\begin{array}{l}\text { Chronischer Reizzustand } \\
\text { Polyvinylchlorid } \\
\text { Hämochromatose } \\
\text { Dioxin (TCDD): Agent Orange }\end{array}$ \\
\hline Bestrahlung & $\begin{array}{l}\text { arsenhaltige } \\
\text { traumatische, durch Unfall verursachte }\end{array}$ \\
\hline Lymphödeme & $\begin{array}{l}\text { posttherapeutische } \\
\text { parasitische (Filariose) } \\
\text { latrogene } \\
\text { Stewart-Treves Syndrom }\end{array}$ \\
\hline Infektion & $\begin{array}{l}\text { Angeborene } \\
\text { Kaposi Sarkom (humaner Herpesvirus 8) }\end{array}$ \\
\hline
\end{tabular}

gleichbar niedrig bei 2,4-3,6 Neuerkrankungen pro 100000 Einwohner pro Jahr [9]. Es gibt insgesamt keine deutliche Geschlechtsprädisposition. Das mittlere Alter aller Patienten bei der initialen Vorstellung beträgt 50-60 Jahre [3].

Ca. $45 \%$ aller Sarkome kommen im Bereich der unteren Extremitäten, $15 \%$ im Bereich der oberen Extremitäten, $10 \%$ in der Kopfund Nackenregion, $15 \%$ im Retroperitonealraum und die restlichen 15\% im Bereich des Abdomens und der Thoraxwand vor [10]. So sind das Muskel-Skelettsystem der Extremitäten und die abdominalen und thorakalen Wände die bevorzugten Manifestationen von Weichgewebesarkomen. Sarkome der Extremitäten treten vorwiegend am Oberschenkel auf (50-60\%).

Während die Ätiologie der meisten Weichgewebesarkome unklar ist, sind einige Risikofaktoren beschrieben ( $\bullet$ Tab. 1). Bis zu $60 \%$ aller Weichgewebesarkome weisen eine somatische Mutation von p53 auf [11]. Auch eine zurückliegende Strahlenexposition kann für die Entstehung von bis zu 5,5\% aller Weichgewebesarkome verantwortlich sein. Das Risiko ist dosisabhängig und die Latenzzeit zwischen Bestrahlung und klinischer Tumormanifestation beträgt ca. 5 Jahre. Das postoperative Grading entspricht bei über $80 \%$ der strahlenassoziierten Fälle gering differenzierten Sarkomen [12]. Zudem kann ein Zusammenhang zwischen dem Kaposi-Sarkom und einer Infektion mit dem humanen Herpesvirus 8 bestehen (HHV8; [13]).

\section{Stadieneinteilung}

Präoperatives Staging und Grading sind Voraussetzungen für die Auswahl des besten Therapieverfahrens. Für Weichgewebesarkome der Extremitäten wird die von der International Union against Cancer (UICC) und von dem American Joint Commitee on Cancer (AJCC) publizierte TNM-Klassifizierung am häufigsten angewendet ( $\odot$ Tab. 2, 3; [14]). Aufgrund ihres atypischen Verhaltens werden Dermatofibrosarcoma protuberans, Kaposi-Sarkom, Desmoidtumor und jedes von Dura, Gehirn, parenchymatösen Organen oder Hohlorganen ausgehende Sarkom nicht eingeschlossen. Wie bei anderen Tumoren schließt das Staging 
Tab. 2 TNM Klassifikation der UICC [10].

\section{TNM-Klassifikation}

TX Primärtumor kann nicht beurteilt werden

T0 kein Primärtumor

T1a Tumor $\leq 5 \mathrm{~cm}$, oberflächlich

T1b Tumor $\leq 5 \mathrm{~cm}$, tief

T2a Tumor $>5 \mathrm{~cm}$, oberflächlich

T2b Tumor $>5 \mathrm{~cm}$, tief

NX regionale Lymphknoten können nicht beurteilt werden

NO keine regionalen Lymphknotenmetastasen

N1 regionale Lymphknotenmetastasen

MX Vorhandensein von Fernmetastasen kann nicht beurteilt werden

M0 keine Fernmetastasen

M1 Fernmetastasen

Tab. 3 Grading-System der UICC [10].

\begin{tabular}{ll} 
& \multicolumn{1}{c}{ Grading-System } \\
GX & histologisches Grading kann nicht beurteilt werden \\
G1 & hoch differenziert, G1 im 3-gradigen System \\
G2 & mäßig differenziert, G1 im 3-gradigen System \\
G3 & schlecht differenziert, G2 im 3-gradigen System \\
G4 & undifferenziert, G3 im 3-gradigen System
\end{tabular}

Tab. 4 Stadienklassifikation nach Enneking [11].

\section{MSTS-Einteilung}

Stadium I-A

Stadium I-B

Stadium II-A

Stadium II-B

Stadium III
G1-2, intrakompartimentaler Tumor, M0

G1-2, extrakompartimentaler Tumor, M0

G3-4, intrakompartimentaler Tumor, M0

G3-4, extrakompartimentaler Tumor, M0

jedes $\mathrm{G}$, jedes T,M1 die präoperative Beurteilung von Tumorgröße, Lymphknotenstatus und das Vorkommen von Metastasen (TNM-Status) sowie das pathologische Grading des Tumors ein. Die in 2010 modifizierte Klassifikation beinhaltet die Veränderung, dass Patienten mit regionalen Lymphknoten jetzt als Stadium III statt IV betrachtet werden [14].

Im klinisch-chirurgischen Bereich ist die MSTS-Einteilung der Musculoskeletal Tumor Society nach Enneking üblich ( $\odot$ Tab. 4; [15]).

\section{Histopathologische Typen}

Die Subtypen der Weichgewebesarkome werden mittels histopathologischer Untersuchungen und zytogenetischer Analysen bestimmt. Bei der Einteilung der malignen Weichgewebetumoren gemäß der aktuellen WHO-Klassifikation von 2002 sind die Zelldifferenzierung und der Malignitätsgrad von großer Bedeutung. Man unterscheidet heute:

- Adipozytische Tumoren (z.B. Liposarkome)

- Fibroblastische/myofibroblastische Tumoren

(z.B. Fibrosarkome)

- Fibrohistiozytische Tumoren

- Glattmuskulär und skelettmuskulär differenzierte Tumoren (z.B. Leiomyosarkome und Rhabdomyosarkome)

- Vaskuläre Tumoren (z.B. Kaposi-Sarkome)

- Perizytische Tumoren

- Chondroossäre Tumoren und

- Tumoren mit ungewisser Differenzierung.
Weichgewebe- und auch Knochensarkome können sich an den Extremitäten durch kontinuierlich ausgedehntes Wachstum lokal ausbreiten. In vielen Fällen bildet der Tumor eine Kapsel, die im Zuge einer radikalen Resektion entfernt werden muss. Eine hämatogene Ausbreitung kommt häufig vor. Bei Tumoren der unteren Extremität werden Fernmetastasen vorwiegend in der Lunge gebildet. Lymphknotenmetastasen treten bei Weichgewebesarkomen mit weniger als $5 \%$ eher selten auf, wie z.B. bei Rhabdomyosarkomen, Angiosarkomen, epitheloidzelligen Sarkomen und Klarzellsarkomen [16,17].

\section{Diagnose}

\section{Anamnese}

Aufgrund ihrer geringen Inzidenz und ihrer Lokalisation in expandierbarem Weichgewebe wird die Diagnose eines Weichgewebesarkoms oft sehr spät oder als Zufallsbefund in Verbindung mit einem anderen medizinischen Problem (z.B. chronisch-venöse Insuffizienz) gestellt. Diese Tumore werden oft vom Patienten in Verbindung mit einem Trauma wahrgenommen und deshalb selbst und vom behandelnden Arzt oft als gutartige Läsion unterschätzt. Meist stellen sich die Patienten mit einer schmerzlosen Schwellung, seltener wegen schmerzhafter Bewegungseinschränkung von Arm oder Bein in einer medizinischen Einrichtung vor. Der durchschnittliche Zeitraum vom Erkennen der initialen Symptome bis zur Vorstellung beim Arzt beträgt für alle Weichgewebesarkome 6 Monate, für Sarkome an den Extremitäten wahrscheinlich weniger [18]. Es liegt immer ein begründeter Verdacht auf einen malignen Weichgewebetumor vor, wenn eine Läsion bei der ersten klinischen Untersuchung größer als $5 \mathrm{~cm}$ ist, sich in tiefer Lage befindet, nach 4 Wochen nicht verschwunden ist, oder Symptome, wie Schmerzen und Parästhesien, zeigt [19].

\section{Klinische Diagnostik}

In der Diagnostik von Weichgewebesarkomen wird zunächst eine gründliche körperliche Untersuchung durchgeführt, die sich nicht nur auf die betroffene Extremität beschränkt sondern den ganzen Körper einschließt. Die zugehörigen Lymphknoten werden ebenfalls untersucht, auch wenn eine lymphatische Ausbreitung bei der Mehrheit der Sarkomtypen ungewöhnlich ist. Der allgemeine Gesundheitsstatus ist in Verbindung mit geplanten Operationen besonders wichtig für Patienten mit akuten oder chronischen Begleiterkrankungen, die das Ausmaß der Resektion und der rekonstruktiven Chirurgie beeinflussen können.

\section{Radiologische Diagnostik}

Eine konventionelle Röntgenaufnahme spielt in der Diagnostik von Weichgewebesarkomen kaum eine Rolle, außer bei Knochensarkomen, wie Osteo- oder Chondrosarkomen.

Die MRT (Magnet-Resonanz-Tomografie)- und/ oder CT (Computer-Tomografie)-Untersuchung stellen dagegen die wichtigsten bildgebenden diagnostischen Methoden für Weichgewebesarkome dar, um eine detaillierte Ausbreitung des lokalen Herdes bzw. die Infiltration in benachbarte Strukturen und Gefäßnervenscheiden sowie mögliche hämatogene und lymphogene Fernmetastasen beurteilen zu können [3]. Mithilfe der Kontrastmittel-Anwendung lassen sich in der MRT vitale und nekrotische Tumoranteile unterscheiden, was für die Auswahl der Biopsielokalisation wichtig ist. 
Die CT ist der MRT überlegen, wenn der Verdacht besteht, dass der Tumor bereits benachbarte knöcherne Strukturen infiltriert oder zerstört hat, da ein Knochen in der MRT kein ausreichendes Signal liefert. Thorakale und abdominale CT sind die Diagnoseverfahren der Wahl für das Staging von „high grade“ Sarkomen und dienen zur Detektion intrapulmonaler und abdominaler Metastasen. Ein bildgebendes Staging regionaler Lymphknoten wird nur beim Synovialsarkom, vaskulärem Sarkom, Rhabdomyosarkom sowie bei epitheloidzelligen und Klarzell-Subtypen empfohlen, bei denen das Risiko einer Lymphknotenbeteiligung zwischen $10 \%$ und $44 \%$ liegt [20].

Eine CT-Angiografie ist eine sinnvolle Methode, um den gesamten Gefäßstatus einer betroffenen Extremität zu bestimmen, eine zugrunde liegende allgemeine Gefäßerkrankung und die Gefäßversorgung des Tumors, die Gefäßinvasion und einen tumorbedingten Verschluss zu klären. Sie kann auch wertvolle Informationen über die Durchführbarkeit mikrovaskulärer Anastomosen und die Gegenwart geeigneter Empfängergefäße, insbesondere bei älteren Patienten, liefern [3].

Die Kombination von morphologischer und funktioneller Information über den Tumorstoffwechsel mit der PET-CT hat in den letzten Jahren im Therapiemanagement von Weichgewebesarkomen an Bedeutung gewonnen.

Die PET-CT wird in ausgewählten Fällen eingesetzt, um die frühzeitige Identifizierung von Lokalrezidiven im postoperativen Verlauf zu bestätigen. Dagegen wird diese diagnostische Methode für das präoperative Staging nicht akzeptiert [21-23].

Die Ultraschalldiagnostik ist eine preiswerte, schnelle und schmerzfreie Methode, die zum Beantworten präoperativer Fragen hilfreich sein kann. Sie hat in der Vergangenheit zu vielen Zufallsbefunden eines Tumors geführt. Die Anwendung des Ultraschalls in der Diagnostik von Weichgewebesarkomen erfordert jedoch gut geschulte Ärzte.

\section{Biopsieverfahren}

Die Grundlage für eine geeignete operative Therapie eines Weichgewebesarkoms bildet die histologische Untersuchung des Tumors. Die histologisch gesicherte Diagnose wird mithilfe von Gewebeproben gestellt, die in den meisten Fällen durch offene Inzisionsbiopsie gewonnen werden. In der anschließenden histologischen Untersuchung wird die histopathologische Typisierung durchgeführt und der Malignitätsgrad bestimmt (Grading).

Repräsentatives Biopsiematerial zu erhalten, ist ein kritischer Schritt, der von einem erfahrenen Chirurgen durchgeführt werden sollte. Vor der Biopsie wird mittels MRT die Tumorgröße bestimmt und die strategische Vorgehensweise geplant.

Die Gewebeentnahme kann entweder durch eine Nadelpunktion erfolgen oder chirurgisch durch Inzisions- oder Exzisionsbiopsie. Die sonografisch- oder CT-gesteuerte Feinnadelbiopsie mit einer Nadelstärke von weniger als einem Millimeter ist eine weit verbreitete sehr sichere Methode mit einer Komplikationsrate unter 1\% [24]. Die Menge des Biopsiematerials ist jedoch sehr klein, reicht häufig nur für eine zytologische Analyse, und die diagnostische Richtigkeit hängt stark von der Erfahrung des Pathologen ab. Bei der Stanzbiopsie mit einer Nadelstärke von mehr als einem Millimeter wird eine größere Gewebemenge mithilfe eines Gewebezylinders entnommen.

Obwohl die Gewebeprobe immer noch zu gering ist, um die umfangreichen histologischen Untersuchungen durchzuführen, hat die Stanzbiopsie den Vorteil, dass Gewebe aus verschiedenen Teilen des Tumors gewonnen wird und so ein umfassenderes
Bild vom Tumor entsteht [25]. Die sonografisch- oder CT-gesteuerte Stanzbiopsie führt daher in bis zu $90 \%$ der Fälle zu einer korrekten Diagnose von Weichgewebesarkomen, eine Feinnadelbiopsie nur in 56 - 76\% der Fälle [25-27]. In mehreren Zentren werden Stanzbiopsien bei operativ nicht resezierbaren Weichgewebesarkomen angewendet (z. B. retroperitoneale Läsionen), um den Gewebetyp zu bestimmen und eine mögliche neoadjuvante Therapie einzuleiten [25].

Der allgemein akzeptierte Standard, um eine sichere präoperative histopathologische Diagnose von Weichgewebesarkomen zu stellen, ist die Inzisionsbiopsie [28]. Bei dieser Methode werden größere Gewebeproben in Verbindung mit einem chirurgischen Eingriff entnommen, wobei der Großteil des Tumors in situ belassen wird. Etwa $2 \mathrm{~cm}^{3}$ Gewebe reichen für eine genaue histologische Beurteilung aus. Wichtig ist die Schnittführung, $3-5 \mathrm{~cm}$ parallel zur Extremitätenachse, damit bei einer späteren Tumorresektion die Narbe und die gesamte Operationshöhle entfernt werden und unnötige Probleme bezüglich der Größe der Defektdeckung vermieden werden können. Die Inzisionsbiopsie ist eine zuverlässige diagnostische Methode und eignet sich für tiefsitzende subkutane oder intramuskuläre Tumoren, die größer als $3-5 \mathrm{~cm}$ sind.

Mit der Exzisionsbiopsie werden kleine Tumoren von weniger als $3 \mathrm{~cm}$ mit einem Sicherheitssaum von $1 \mathrm{~cm}$ vollständig entfernt und stehen für die histologische Untersuchung zur Verfügung [28].

Bei einem relativ neuen Biopsieverfahren, der Vakuumsaugbiopsie, wird mehr Gewebematerial für histologische Untersuchungen gewonnen als bei anderen konventionellen Nadelbiopsien. Dadurch ist die Vakuumsaugbiopsie in Bezug auf das Grading zuverlässiger und wird zunehmend eingesetzt [28].

\section{Chirurgische Therapie}

Art und Zeitpunkt des chirurgischen Eingriffs werden von der multidisziplinären Tumorkonferenz bestimmt, sobald die Ergebnisse aller diagnostischen Verfahren, der radiologischen Bildgebung und der Histologie vorliegen. Jede Diagnose muss durch eine Referenzpathologie überprüft werden, weil aufgrund der Seltenheit und zahlreicher Untergruppen von Weichgewebesarkomen und nicht selten unzureichendem und nicht repräsentativem Biopsiematerial eine exakte Diagnosestellung erschwert wird. So konnte zwischen der Erstdiagnose und nachfolgender referenzpathologischer Untersuchung eine deutliche Diskrepanz in bis zu 70\% der Fälle festgestellt werden [29]. Von besonderer Bedeutung für die Therapieentscheidung bei malignen Tumoren ist zudem das histologische Grading, das mit dem Metastasierungsrisiko korreliert und so als wichtiger Prognoseparameter gilt [28].

Das vorrangige Ziel der Operation von Weichgewebesarkomen der unteren Extremität ist das vollständige Entfernen des Tumors, da dies am ehesten eine komplette Heilung in Aussicht stellt. Das sekundäre Ziel, ein Bein oder einen Fuß so weit wie möglich zu erhalten, wird durch die Integration plastisch-rekonstruktiver Operationsverfahren meistens erreicht. Für die untere Extremität sind dabei Stabilität und Belastbarkeit wichtiger als die funktionelle Mobilität und Sensibilität. Welche operativen Methoden zur Anwendung kommen, hängt auch von anderen Faktoren ab, wie von patientenspezifischen Daten, z.B. Alter, Größe und Gewicht sowie vorbestehenden Begleiterkrankungen (z.B. arterielle Verschlusskrankheit, Diabetes mellitus und Venenerkrankungen), Medikamenteneinnahme, früheren Operationen, funktionellen und ästhetischen Ansprüchen sowie von den verfügbaren rekonstruktiven Optionen. 


\section{Tumorresektion}

Die weite bzw. radikale Resektion mit ausreichendem Sicherheitsabstand ist heute das Standardverfahren für die Resektion von Weichgewebesarkomen der Extremitäten. Tumorpositive Resektionsränder können das Risiko für Lokalrezidive erhöhen und schließlich die Überlebensrate vermindern [30].

Es gibt keine überzeugenden Studien über exakte onkologisch ausreichende Sicherheitsabstände, aber viele Zentren empfehlen einen Sicherheitsabstand von $2 \mathrm{~cm}$ in die Tiefe und von $4-5 \mathrm{~cm}$ zur Seite $[18,31,32]$. Diese Resektionsgrenzen können in der Peripherie der unteren Extremität aus anatomischen Gründen nicht immer eingehalten werden $[3,33]$. Einige Autoren schlagen deshalb einen Sicherheitsabstand von mindestens $1 \mathrm{~cm}$ vor, wenn nicht große Nerven beteiligt sind [26,34]. Zudem zeigen retrospektive Studien für knappe R0-Resektionen mit Sicherheitsabständen von über $1 \mathrm{~mm}$ und begleitender adjuvanter Radiatio die Effektivität des Verfahrens mit Lokalrezidivraten um 10\% [34-36]. Nach Recherche der Literatur empfiehlt die Gruppe um Steinau und Mitarb. für die klinische Praxis in Verbindung mit der Radiatio einen Sicherheitsabstand von 1-2 cm [33].

Das praktische Vorgehen bei der Tumorresektion wurde durch Enneking wesentlich beeinflusst, der das Ausmaß der Resektion und die Resektionsgrenzen nach dem biologischen Wachstumsverhalten der Weichgewebesarkome richtete, das durch das histopathologische Grading, die lokale Tumorausdehnung und Metastasierung bestimmt wird [37]. Da die Weichgewebesarkome hauptsächlich in ihren Kompartimenten in longitudinaler Ausbreitung wachsen, schlug er eine Kompartmentresektion vor, bei der alle das Kompartiment seitlich begrenzenden Gewebe, also auch der Ursprung und der Ansatz des Kompartiments, mit entfernt werden. Nach Enneking spielen bei der weiten Resektion sichere biologische Grenzschichten, wie Muskelfaszien, Periost, Gelenkkapsel, Perineurium und Adventitia eine größere Rolle als ausreichende Sicherheitsabstände zum gesunden Gewebe. Die radikale Resektion großer Kompartimente verursacht beträchtliche Gewebedefekte und kann größere operative Rekonstruktionen für den Patienten zur Folge haben. Diese Kompartmentresektion wird heute noch für Hochrisiko-Sarkome favorisiert [38].

Der Chirurg sollte den Tumor während des Eingriffs weder berühren noch sehen (sog. „No Touch-Technik“). Außer dem Tumor werden in longitudinaler Richtung 3-4 cm Weichgewebe (Muskulatur) bzw. die Sehne oder der Ansatz des Kompartiments mit angrenzendem Knochen mit reseziert [39].

Chirurgische Tumorverletzungen sind $\mathrm{zu}$ vermeiden, weil sie durch die Kontamination des gesunden umgebenden Gewebes mit Tumorgewebe mit einer erheblichen Abnahme der 5-Jahres-Überlebensraten verbunden sind ( $47 \%$ vs. $87 \%$ in einer Studie; [40]). Um einen Blutverlust und eine mögliche Kontamination durch Tumorzellverschleppung zu vermindern, ist eine Blutsperre anzulegen oder eine vorübergehende Okklusion der großen Gefäße (iliakale und femorale Arterien) mittels Klemmen anzustreben. Weiterhin sollten frühere Hautinzisionen bzw. Biopsiezugänge mit einem Abstand von möglichst bis zu $4 \mathrm{~cm}$ weit umschnitten werden und eine inzisionsnahe RedonAusleitung im Wundwinkel erfolgen. Es muss gewährleistet sein, dass die Redondrainage bei späteren Operationen mit entfernt werden kann.

Eine regelmäßige regionale Lymphknotendissektion ist bei Weichgewebesarkomen nicht indiziert, da nur wenige Sarkomtypen Lymphknotenmetastasen aufweisen. Die Rolle von Sentinel-Lymphknoten in Hochrisiko-Subtypen von Weichgewebesarkomen ist bisher ungeklärt $[41,42]$.
Mithilfe präoperativer radiologischer Methoden (MR-Angiografie oder CT-Angiografie) kann die Lage von Gefäßen und Nerven zum Tumor bestimmt und das chirurgische Vorgehen bezüglich beteiligter und benachbarter Gefäße und Nerven geplant werden. Im Fall einer Gefäßinfiltration oder einem tumorbedingtem Gefäßverschluss müssen die betroffenen in den Tumor eingebundenen Gefäße reseziert werden. Um die arterielle Durchblutung der unteren Extremität nach Tumorentfernung wiederherzustellen, müssen Hauptarterien möglichst sofort durch Veneninterponate oder Gefäßprothesen rekonstruiert werden. Venentransplantate für die Rekonstruktion großer Venen sollten wegen des Thromboserisikos nur für ausgewählte Patienten verwendet werden.

Wenn tiefe volumenstarke Venen in der Nähe des Tumors reseziert werden müssen, sollten oberflächliche größere Venen mit ihren subkutanen Abflussgebieten möglichst erhalten werden, um die Gefahr von Lymphödemen zu minimieren. Während der Präparation gelingt es häufig, kleine Muskel- oder Hautgefäße zu erhalten, welche die Wundheilung unterstützen und als Anschlussstellen für Hautlappenplastiken dienen.

Die gleiche Dissektionsstrategie wird auch auf große Nervenstrukturen an der unteren Extremität angewendet. Alle vom Tumor eingeschlossenen Nerven müssen reseziert werden. Kleinere Nervenstrukturen sollten erhalten werden, um motorische Funktionen zu verbessern.

Maligne Weichgewebesarkome wachsen relativ häufig in der Nähe von Knochen, eine Arrosion oder Infiltration des Knochens findet man dagegen sehr selten. Bei knochennahen Sarkomen verbleibt das Periost als Grenzschicht am Resektat.

Eine Tumornachresektion nach auswärtiger Voroperation ist onkochirurgisch schwierig. Da in einem solchen Fall makroskopische Tumorreste im Bereich des ursprünglichen Sarkoms vermutet und eine iatrogene Tumorzellverteilung im eröffneten Gewebe erwartet werden kann, ist aus onkologischer Sicht ein radikaler operativer Eingriff erforderlich [39]. Das chirurgische Vorgehen entspricht daher einer weiten gegebenenfalls kompartiment-orientierten Resektion. Im Vorfeld sollte der histologische Tumorbefund durch einen eigenen Pathologen bestätigt und das gesamte Behandlungskonzept in der interdisziplinären Tumorkonferenz besprochen werden. Bei einem G2-/G3-Sarkom mit radiologisch nachgewiesenem Resttumor ist eine Vorbehandlung sinnvoll. Bei der anschließenden operativen Revision müssen alle durch die Vorbehandlung potenziell kontaminierten Gewebe reseziert werden. Daraus resultiert ein größerer Gewebedefekt mit aufwendigen plastischen Rekonstruktionen [39].

\section{Rekonstruktionsverfahren}

Ziel der Rekonstruktion ist ein größtmöglicher Funktionserhalt der Extremität und ein ästhetisch zufriedenstellendes Ergebnis. Im Bereich der unteren Extremität ist die Belastbarkeit wichtiger als die Mobilität und der Erhalt der Sensibilität in Bein und Fuß weniger relevant als im Bereich der oberen Extremität. Jeder Wundverschluss mit plastischer Defektdeckung dient dazu, große Hautdefekte nach Sarkomresektion abzudecken und exponierte Knochenvorsprünge, Gelenke oder Amputationsstümpfe zu bedecken. Eine suffiziente Weichteilwiederherstellung mit spannungsfreiem Wundverschluss führt zudem zu weniger Wundheilungsstörungen nach postoperativer Radiatio.

Der Primärverschluss ist abhängig von der Tumorgröße und der Lokalisation. Am Oberschenkel ist nach Sarkomresektion häufig ein Primärverschluss möglich, während diese Methode zum Wundverschluss im Bereich des Knies und am Unterschenkel gewöhnlich nicht angewendet werden kann [3]. Der Primärver- 

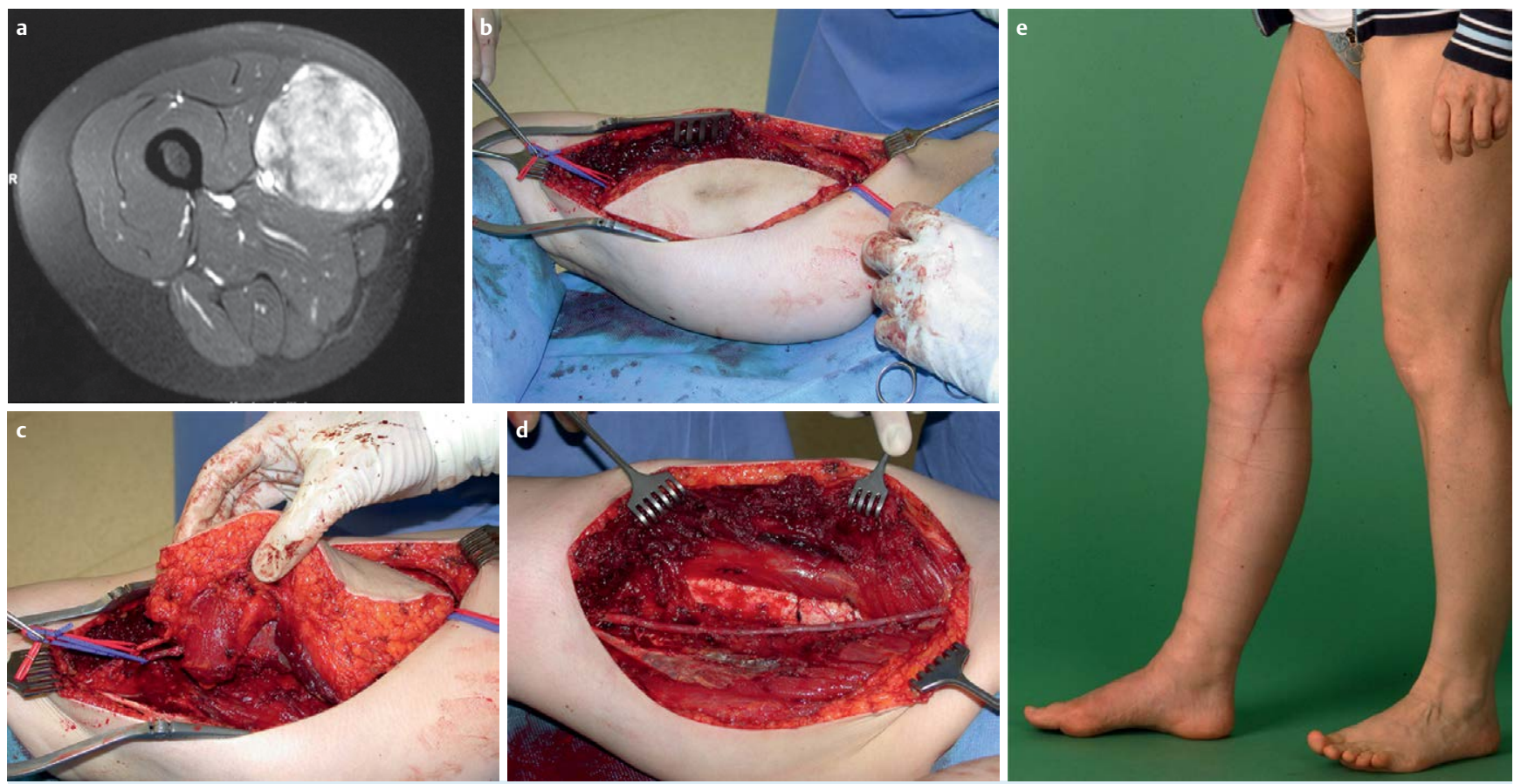

schluss sollte mehrschichtig und möglichst spannungsfrei erfolgen mit besonders sorgfältigen Subkutan- und Hautnähten ( $\bullet$ Abb. 1). Auf diese Weise werden die Serom- und Hämatombildung und Dehiszenzen sowie Wundheilungsstörungen vermieden, die den Beginn einer adjuvanten Radiotherapie verzögern können.

Wenn ein Primärverschluss nicht infrage kommt, ist gegebenenfalls die Verlagerung benachbarten Gewebes möglich, d.h. die spannungsfreie Abdeckung kleiner Defekte mit fasziokutanen Lappenplastiken. Vorteile dieser Methode sind die geringe Hebemorbidität und die geringe Belastung für den Patienten.

Eine Spalthauttransplantation wird verwendet wenn sich der Defekt auf die Oberfläche beschränkt, ein gut perfundiertes Wundgewebe vorliegt und keine Knochen, Knorpel, Sehnen, Nerven oder Blutgefäße freiliegen. Vorteile dieser Technik sind die geringe Hebemorbidität und schnelle Durchführbarkeit. Jedoch ist Spalthaut vulnerabel und strahlenempfindlich.

Zum Decken von Defekten im Oberschenkelbereich haben sich gestielte Lappenplastiken (fasziokutane Lappen und Muskellappen mit oder ohne Hautinsel aus dem tiefen unteren Oberbauch-Arteriensystem des unteren Abdomens oder aus dem Glutealbereich) bewährt ( $\bullet$ Abb. 2). Vorteile dieser Lappenplastiken sind die sichere Durchblutung über den Gefäßstiel und die 

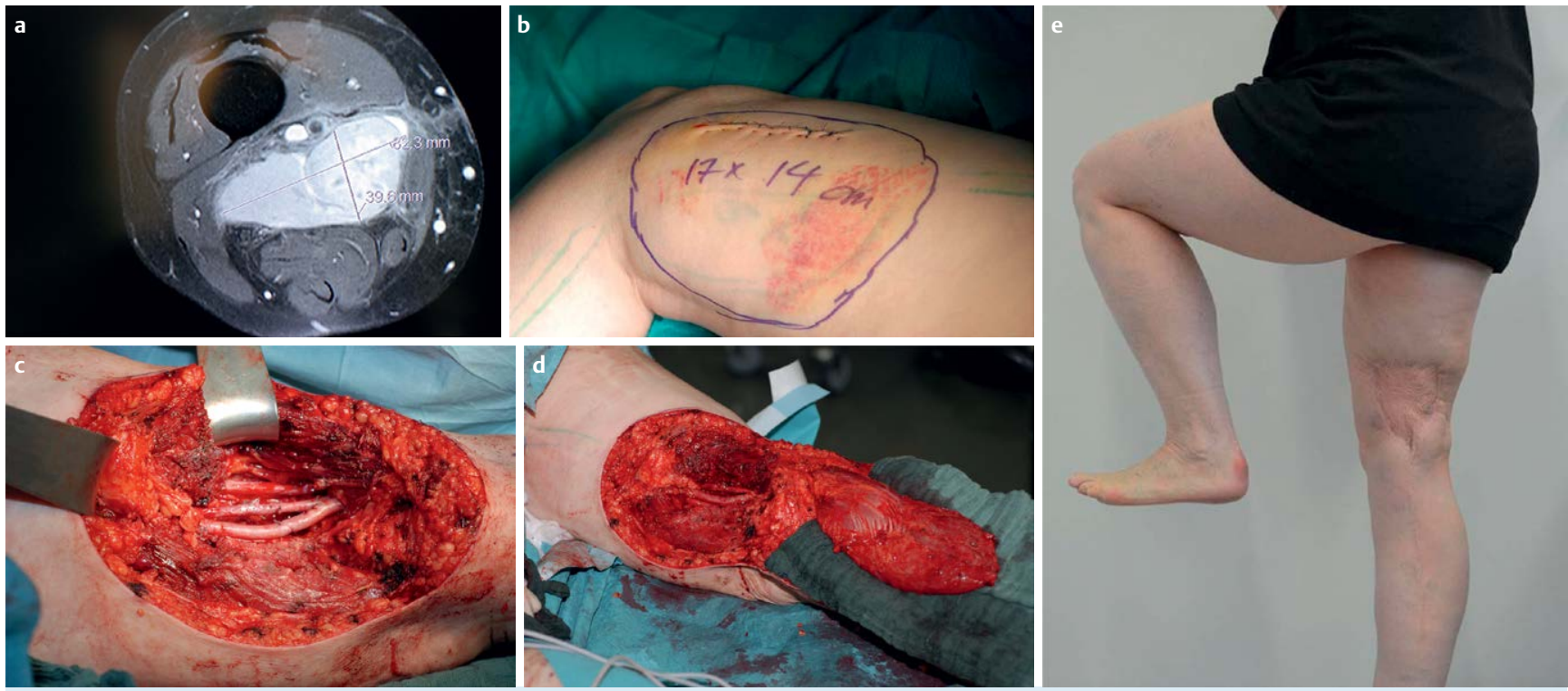

Abb. 3 a 55-jährige Patientin mit undifferenziertem pleomorphem Sarkom im Bereich der rechten Kniekehle. b Zustand nach auswärts erfolgter Inzisionsbiopsie: es erfolgt die „wide excision“. c Die Arteria und Vena poplitea sowie der Nervus tibialis konnten nach entsprechendem Stripping-Technik erhalten werden. d Die Defektdeckung erfolgte mittels proximal gestieltem medialem Gastrocnemiuslappen, das Bild zeigt den gehobenen Muskellappen. e Ergebnis 3 Jahre nach Operation.

suffiziente Dicke. Allerdings können bei verschiedenen Lappenentnahmestellen funktionelle und ästhetische Einschränkungen zurückbleiben.

Für Defektdeckungen am Oberschenkel, am Knie, im Poplitealbereich und am proximalen Unterschenkel kommt häufig der M. gastrocnemius zur Anwendung, der voluminös genug ist, um größere Defekte abzudecken ( $\bullet$ Abb. 3). Zudem kann der sehnige Anteil zur Rekonstruktion von Bändern und Sehnen dienen. Der Kraftverlust für die Beugung im Sprunggelenk beläuft sich auf $20 \%$ [43], der Hebedefekt ist gering. Für Defektdeckungen am Unterschenkel und Fuß kommt u.a. auch der Suralislappen infrage. Dieser fasziokutane Lappen gilt bei Patienten mit guter Durchblutungssituation am Unterschenkel als relativ sicheres Verfahren. Vorteil des Suralislappen ist die einfache Präparation, der Nachteil sind häufige venöse Abstromprobleme [43].

Wenn gestielte Lappenplastiken nicht ausreichen oder das zu transplantierende Gewebe mit dem zugehörigen Gefäßstiel durch Voroperationen oder Bestrahlung geschädigt wurde, sind freie Lappenplastiken indiziert. Bei großen Defekten kommt die Latissimus-dorsi-Lappenplastik zur Anwendung. Alternativ wird die Anterolateral-Thigh-Lappenplastik (ALT) verwendet. Für die untere Extremität kommen nach Weichgewebesarkom-Resektion auch häufig der Radialis-, Paraskapular- und Grazilislappen zum Einsatz ( $\odot$ Tab. 5, $\odot$ Abb. 4). Eine Unterart der gestielten und freien Lappenplastiken stellen die Compound-Lappen dar, die in einem Transplantat mehrere Gewebearten kombinieren (Composite-Lappen, Chimärenlappen).

Mithilfe mikrochirurgischer Techniken können auch aus Radikalitätsgründen resezierte Knochen mit und ohne Weichgewebe verpflanzt werden. Breite Anwendung finden der Skapulaknochen in Verbindung mit einer Latissimus-dorsi-Lappenplastik und die freie mit Hautinsel, die zur Stabilisierung der distalen Tibia transplantiert wird. Auch Kombinationstechniken, bei denen eine vaskularisierte Fibula in ein knöchernes Allotransplantat eingebracht wird [44], haben viel versprechende Resultate bei nach Resektion eines Weichgewebesarkoms zusätzlich
Tab. 5 Häufig verwendete freie und gestielte Lappen für die untere Extremität.

A. Freie Lappenplastiken durch Defektdeckung an der
unteren Extremität
Latissimus-dorsi-Lappen
Anterolateraler Oberschenkel-Lappen
Radialis-Lappen
Paraskapular-Lappen
Grazilis-Lappen
lateraler Oberarmlappen
B. Gestielte Lappenplastiken durch Defektdeckung an der
unteren Extremität
Vertikaler rectus-abdominis-myocutaner Lappen (VRAM)
Perforatorlappen
Gastrocnemius
Suralislappen
Propellerlappen

vorhandenen ausgedehnten Skelettdefekten gezeigt [45], - Abb. 5, 6.

Hauptsächlich bei jungen Patienten werden nach großen Sarkomresektionen auch Nerven transplantiert, insbesondere der N. suralis. Dabei wird die End-zu-Seit-Naht von Nerventransplantaten an intakte Nerven zur schnelleren Reinnervation bevorzugt.

Primäre Amputationen für die Behandlung von Sarkomen der unteren Extremität sind bei weniger als 5\% aller Patienten und weniger als $15 \%$ aller Rezidive notwendig, wobei sie ein vergleichbares Langzeitüberleben zeigen [18,31,47]. Die Indikation zur Amputation ist dann zu erwägen, wenn ein Weichgewebesarkom die Membrana interossea der unteren Extremität durchbricht, schon eine lokoregionale Ausbreitung zeigt oder sehr große ulzerierte Rezidivtumore aufweist.

Auch wenn chronische Begleiterkrankungen, wie chronische Veneninsuffizienz oder arteriosklerotische Verschlusskrankheit, eine Tumorentfernung mit tumorfreien Resektionsrändern und 

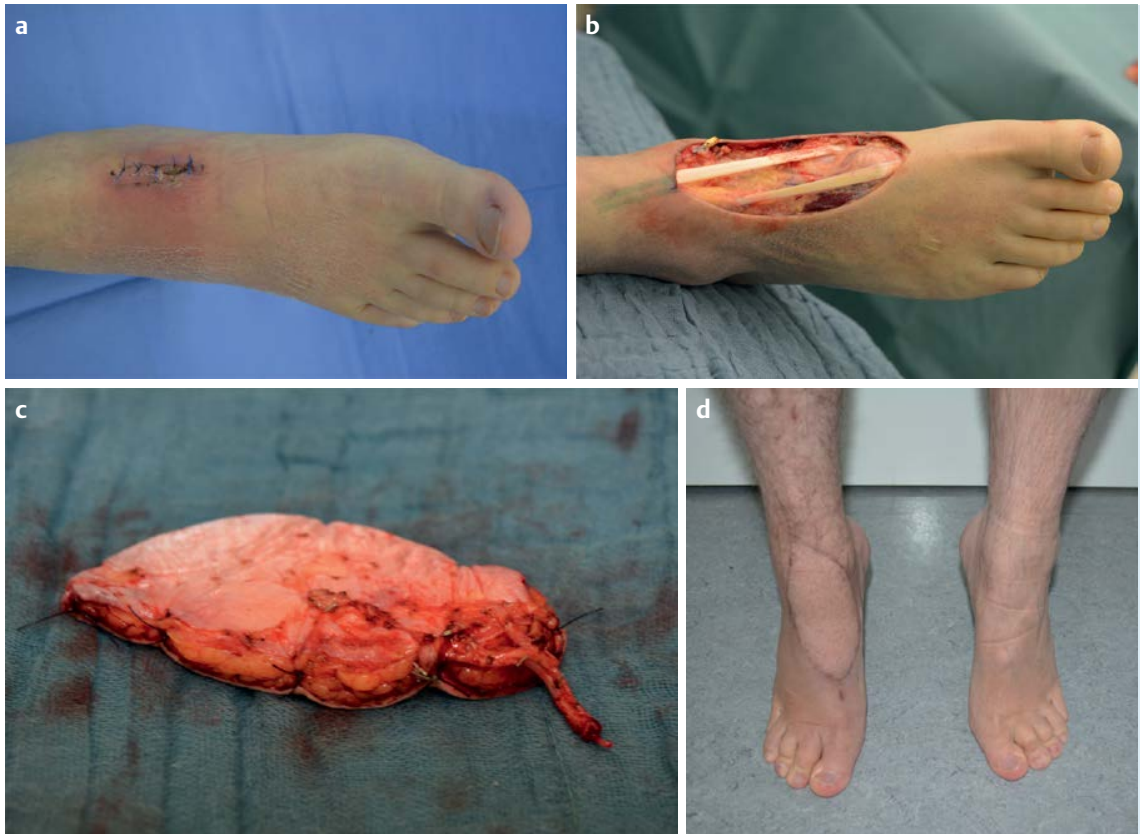

Abb. 4 a Zustand nach auswärtiger Exzisionsbiospie eines myxofibroiden Sarkomes des rechten Fußrückens bei einem 47-jährigen Patienten. b Operationssitus nach radikaler Tumornachresektion. c Ein anterolateraler Oberschenkellappen (ALT) wurde zum optimalen Gleiten der exponierten Sehnen als adipofasziokutanes Transplantat präpariert. d Funktionelles Ergebnis mit ästhetisch ansprechendem Resultat 8 Monate post operationem.
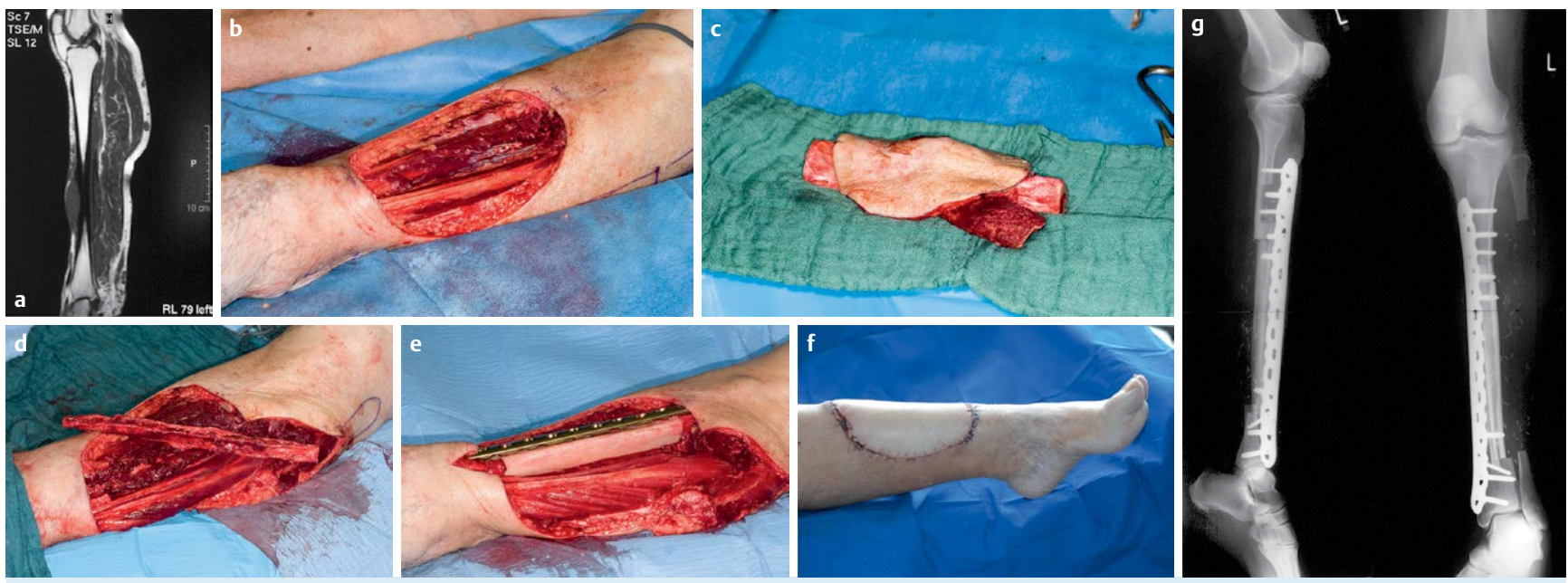

Abb. 5 a Zweites Rezidiv eines pleomorphen Sarkoms des linken Unterschenkels bei einer 65-jährigen Patientin mit Osteolyse der Tibia (MRT).

b, $\mathbf{c}$ Intraoperativer Situs mit Resektion von $16 \mathrm{~cm}$ Tibia einschließlich Tumor und Weichteilgewebe. $\mathbf{d}$ Knochenrekonstruktion mit ipsilateral gestielter Fibula und Allograft. e Osteosynthese durch winkelstabile Platte und Allograft (Technik nach Capanna R, et al. [44]). f Defektdeckung mittels freiem ALT-Lappen. g Röntgenverlaufskontrolle 6 Monate post operationem mit zunehmender knöcherner Durchbauung.

eine sofortige Defektrekonstruktion ausschließen, kann eine Amputation unumgänglich sein.

Eine Alternative dazu stellt eine Segmentamputation dar, bei der nach Tumorresektion der unversehrte distale Extremitätenteil mittels Osteosynthese mit dem proximalen Extremitätenteil verbunden wird. Bei der sog. Borggreve-Plastik wird der Unterschenkel um $180^{\circ}$ verdreht mit dem Oberschenkel osteosynthetisch verbunden (Umkehrplastik), sodass sich die Ferse auf Kniehöhe befindet und dadurch die Prothesenversorgung verbessert wird [43].

\section{Ergebnis und Chemotherapie}

Die Radiatio ist heute die wichtigste zusätzliche Behandlungsmodalität in der Therapie von Weichgewebesarkomen. Eine präoperative (neoadjuvante) Strahlentherapie bei großen Tumoren führt zu einer Tumorverkleinerung (Downstaging) und damit zu einer besseren Resektabilität. Zudem reduziert sie eine potenzielle Tumoraussaat. Nachteile der präoperativen Bestrah- lung im Vergleich zur postoperativen (adjuvanten) Radiotherapie sind eine höhere Rate von Wundheilungsstörungen, die mikrochirurgische Rekonstruktionen wegen der schlechteren Gewebequalität beeinträchtigen.

Die prä- und postoperative Chemotherapie wird bei Patienten mit lokal fortgeschrittenen Weichgewebesarkomen angewendet. Die Rolle beider Therapieformen wird in klinischen Studien (EORTC, COSS, EURO-Ewing) untersucht. Durch Chemotherapien werden mikrochirurgische Rekonstruktionsverfahren nicht negativ beeinflusst. Gegebenenfalls werden Kinder mit moderner Chemotherapie zunächst neoadjuvant behandelt. Besonders die neoadjuvante isolierte Extremitätenperfusion mit TNF alpha und Melphalan gilt heute als eine leistungsstarke Möglichkeit der Tumorreduktion.

\section{Ergebnis und Prognose}

Um das Outcome von Patienten mit Weichgewebesarkomen an den Extremitäten zu optimieren, ist es hilfreich, solche Patienten 

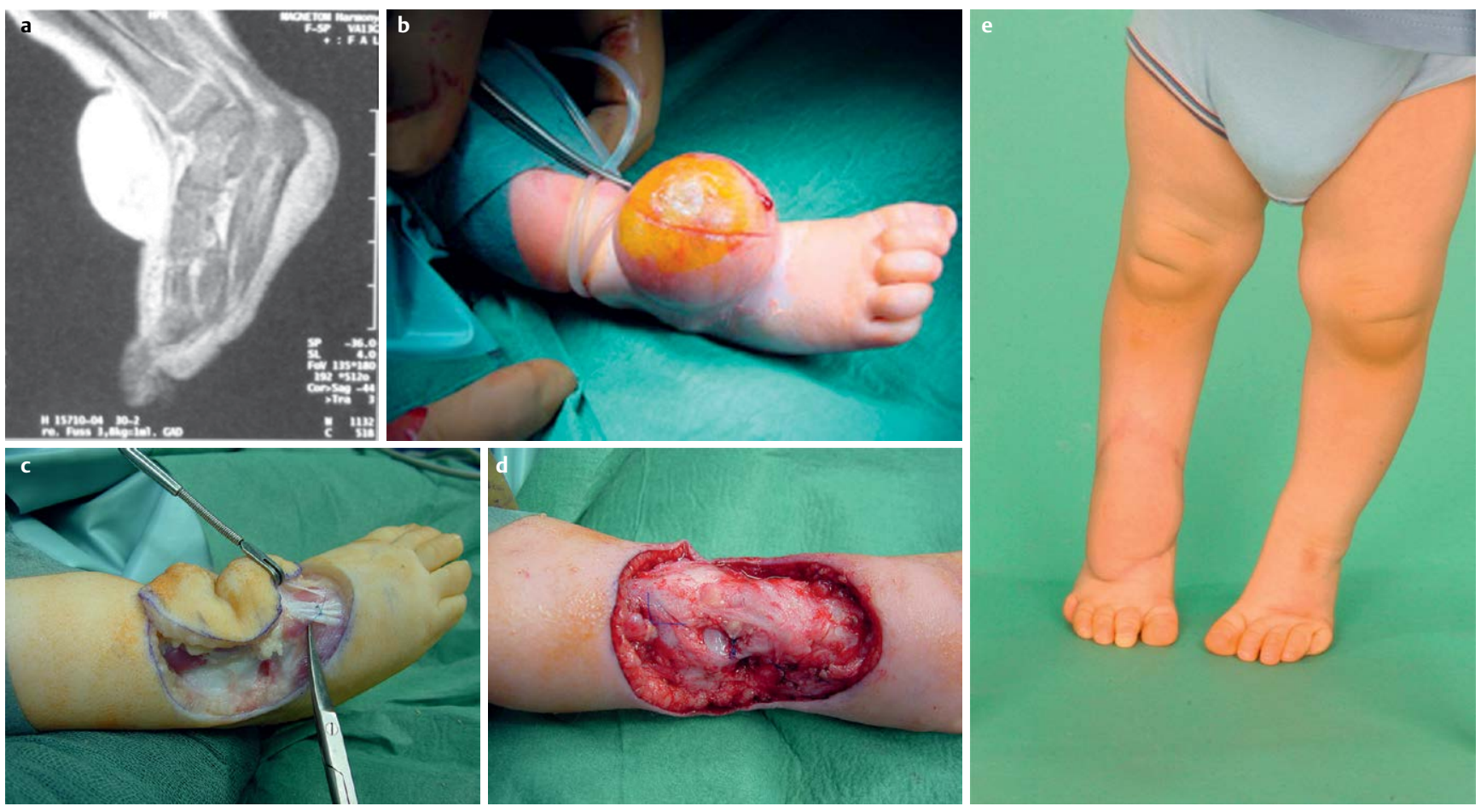

in spezialisierten Zentren nach einem multimodalen Konzept zu behandeln. Ähnlich wie bei anderen seltenen malignen Tumoren, hat sich gezeigt, dass die Behandlung durch erfahrene Chirurgen sowie die Einbindung des Patienten im Rahmen seiner Erkrankung in ein Tumorboard das Ergebnis bzw. die Prognose verbessert [48]. Primäre Resektion und Rekonstruktion sind als voneinander abhängige Komponenten des Behandlungskonzepts gemeinsam zu planen, um die Lokalrezidivrate, das Überleben oder die Funktion der Extremität nicht zu beeinträchtigen oder zu gefährden. Eine Rekonstruktion im Zuge der Tumorresektion führt zu deutlich geringeren Komplikationsraten und verkürzt die Heilung gegenüber sekundären Eingriffen [49].

Wichtige Prognosefaktoren von Weichgewebesarkomen sind neben bestimmten histopathologischen Subtypen auch Tumortiefe, Tumorgröße, Tumorgrading, Lokalisation (proximal vs. distal), Resektionsränder, Patientenalter und Gesundheitsstatus bei Vorstellung. Daraus ergeben sich für diese heterogene Gruppe maligner Erkrankungen sehr unterschiedliche Prognosen. Die aktuelle altersangepasste Todesrate für Weichgewebesarkome, die auf Daten von 2001 bis 2005 basiert, liegt bei 1,3 pro 100000 pro Jahr [2].

Es liegen relativ wenige Untersuchungen zur gesundheitsbezogenen Lebensqualität von Patienten mit Weichgewebesarkomen der unteren Extremität vor. Eine deutsche Studie verglich die Lebensqualität (EORTC QLQ-C30) von 124 Patienten, die von 1980-2000 entweder extremitätenerhaltend operiert oder amputiert wurden [50]. Nach einer mittleren Nachbeobachtungszeit von 46 Monaten waren die funktionellen Ergebnisse (MSTSScore) nach Extremitätenerhalt tendenziell besser, während sich keine Unterschiede in der Lebensqualität zeigten.

\section{Diskussion}

$\nabla$

Die Prognose von Patienten mit Weichgewebesarkomen an den Extremitäten hat sich in den letzten 20 Jahren nicht relevant verändert. Das zeigt eine große amerikanische Studie mit 1706 Patienten, die von 1997 bis 2001 aufgrund von Weichgewebesarkomen an den Extremitäten behandelt und im Durchschnitt 55 Monate nachbeobachtet wurden [2]. Das krankheitsabhängige 5-Jahres-Überleben des gesamten Patientenkollektivs von $85 \%$ und das krankheitsabhängige 5-Jahres-Überleben von Hochrisi- 
kopatienten von $61 \%$ unterscheiden sich nicht signifikant von entsprechenden Überlebensparametern früher behandelter Patienten. Laut einer aktuellen Studie weist die G2/G3-Gruppe von Weichgewebesarkomen eine 5-Jahres-Überlebensrate von 50 bis $60 \%$ auf, während die G1-Tumoren eine 5-Jahres-Überlebensrate von $90 \%$ zeigen [51].

Bei 85 Patienten mit Weichgewebesarkomen an der unteren Extremität, die von 1980 bis 1996 extremitätenerhaltend operiert und mit Lappenplastiken versorgt wurden, findet sich eine 5-Jahres-Überlebensrate von 62\% [18]. Bei 18 dieser Patienten wurden wegen lokaler Knocheninfiltration besondere Knochentransplantationen, konventionelle partielle Fußamputationen oder Hinterfuß-Amputationen durchgeführt. Orthopädische Schuhe mit Einlagen führten hier zu einem besseren Gangbild als vergleichbare Prothesen für das untere Bein.

Eine finnische Studie untersuchte 73 Patienten mit Weichgewebesarkom an der unteren Extremität, bei denen mikrochirurgische Gewebeverpflanzungen erfolgten mit einer Nachbeobachtungszeit von 66 Monaten [52]. Drei Viertel der Patienten waren in der Lage normal zu gehen oder hatten nur geringe Gehbeeinträchtigungen. Für alle Patienten ergaben sich ein krankheitsabhängiges Gesamtüberleben von $70 \%$, ein rezidivfreies 5-Jahres-Überleben von $82 \%$, ein metastasenfreies Überleben von $59 \%$ sowie ein krankheitsfreies Überleben von $56 \%$.

In einer großen deutschen Studie wurden 167 Patienten mit Liposarkomen an den Extremitäten mit einer mittleren Nachbeobachtungszeit von 36 Monaten untersucht [32]. Die Autoren geben ein 5-Jahres-Gesamtüberleben von $79 \%$ an.

\section{Fazit}

\section{$\nabla$}

Eine optimale Behandlung von Patienten mit Weichgewebesarkomen sollte nur in hochspezialisierten Zentren erfolgen, wo eine interdisziplinäre Zusammenarbeit im Rahmen der multimodalen Therapie und eine möglichst kurative, onkologisch radikale Tumorresektion flankiert von extremitätenerhaltenden Maßnahmen durch plastisch-chirurgische Operationsverfahren sichergestellt sind, die die Prognose der Patienten entscheidend verbessern.

Hierdurch kann in mehr als 95\% der Fälle eine radikale Tumorexzision durchgeführt werden und gleichzeitig auch große Defekte verschlossen sowie die Funktion der Extremität erhalten werden.

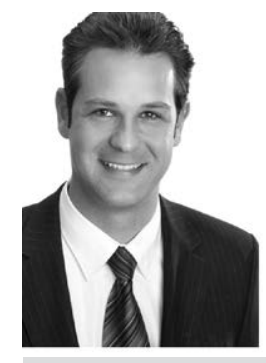

Torsten Schloßhauer

geboren am 01.03.1979 in Kassel. Studium der Humanmedizin an der Philipps Universität Marburg, Praktisches Jahr am Kantonsspital Luzern (Frau Prof. Dr. V. Briner) sowie in der Klinik für Plastische, Ästhetische und Rekonstruktive Chirurgie Rotes Kreuz Krankenhaus Kassel (Prof. Dr. E.M. Noah). 2007 Approbation als Arzt. Promotion mit dem Abschluss „magna cum laude“ an der Universität Marburg. 2007-2012 Chirurgische Weiterbildung unter Prof. M. Rothmund und Prof. D. Bartsch am Universitätsklinikum Marburg, Rotation in die Klinik für Unfall-, Hand- und Wiederherstellungschirurgie des Universitätsklinikums Marburg (Prof. Dr. S. Ruchholtz). 2012-2014 Assistenzarzt in der Abteilung für Plastische
Chirurgie, Handchirurgie, Zentrum für Schwerbrandverletzte des Universitätsklinikums Schleswig-Holstein, Campus Lübeck (Prof. Dr. P. Mailänder). 2013 Facharzt für Allgemeinchirurgie. Seit 07/2014 Tätigkeit in der Abteilung für Plastische, Hand- und Rekonstruktive Chirurgie der Berufsgenossenschaftlichen Unfallklinik Frankfurt am Main, Klinik für Plastische Chirurgie des Universitätsklinikums Frankfurt (Prof. Dr. med. Dr. med. habil. M. Sauerbier).

\section{Interessenkonflikt: Nein}

\section{Literatur}

1 Clark MA, Fisher C, Judson I et al. Soft tissue sarcomas in adults. New Engl J Med 2005; 353: 701-711

2 Weitz J, Antonescu CR, Brennan MF. Localized extremity soft tissue sarcoma: improved knowledge with unchanged survival over time. J Clin Oncol 2003; 21: 2719-2725

3 Giessler GA, Sauerbier M. Lower extremity sarcoma reconstruction. In: Neligan PC, Song DH, Van Beek AL. Plastic Surgery, Lower Extremity, Trunk and Burns. 3. Aufl. London, New York, Oxford, St. Louis, Sydney, Toronto: Elsevier, 2013; 4: 101-126

4 Müller M, Bickert B, Germann $G$ et al. Soft-tissue sarcoma of the forearm and hand. Chirurg 2008; 79: 682-688

5 Vetter M, Germann G, Bickert B et al. Current strategies for sarcoma reconstruction at the forearm and hand. J Reconstr Microsurg 2010; 26: $455-460$

6 Steinau HU, Daigeler A, Langer $S$ et al. Limb salvage in malignant tumors. Semin Plast Surg 2010; 24: 18-33

7 Megerle K, Sauerbier M. Reconstructive treatment of soft tissue sarcoma of the upper extremity. J Hand Surg Am 2011; 36: 1241-1247

$8 \mathrm{Zahm} \mathrm{SH,} \mathrm{Fraumeni} \mathrm{JF.} \mathrm{The} \mathrm{epidemiology} \mathrm{of} \mathrm{soft} \mathrm{tissue} \mathrm{sarcoma.} \mathrm{Sem}$ Oncol 1997; 24: 504-514

9 www.seer.cancer.gov

10 Enzinger FM, Weiss SW. Soft Tissue Tumors. 3. Aufl. St Louis: Mosby, 1995

11 Das P, Kotilingam D, Korchin B et al. High prevalence of p53 exon 4 mutations in soft tissue sarcoma. Cancer 2007; 109: 2323-2333

12 Brady MS, Gaynor JJ, Brennan MF. Radiation-associated sarcoma of bone and soft tissue. Arch Surg 1992; 127: 1379-1385

13 Chang YI, Cesarman MS, Pessin F et al. Identification of herpesvirus-like DNA sequences in AIDS-associated Kaposi's sarcoma. Science 1994; 266: $1865-1869$

14 Wittekind C, Meyer HJ. TNM-Klassifikation maligner Tumoren. 7. Aufl. Weinheim: Wiley-VCH, 2010

15 Enneking WF, Spanier SS, Goodman MA. A system for the surgical staging of musculoskeletal sarcoma. Clin Orthop Relat Res 1980; 153: $106-120$

16 Fong Y, Colt DG, Woodruff JM et al. Lymph node metastasis from soft tissue sarcoma in adults. Analysis of data from a prospective database of 1772 sarcoma patients. Ann Surg 1993; 217: 72-77

17 Daigeler A, Kuhnen C, Moritz $R$ et al. Lymph node metastases in soft tissue sarcomas: a single center analysis of 1597 patients. Langenbecks Arch Surg 2009; 394: 321-329

18 Steinau HU, Homann HH, Drucke D et al. Resection method and functional restoration in soft tissue sarcomas of the extremities. Chirurg 2001; 72: 501-513

19 Johnson CJ, Pynsent PB, Grimer RJ. Clinical features of soft tissue sarcoma. Ann R Coll Surg Engl 2001; 83: 203-205

20 Stroszczynski C, Kittner T. Radiologische Diagnostik. In: Schlag PM, Hartmann JT, Budach V. Weichgewebetumoren - Interdisziplinäres Management. Berlin, Heidelberg: Springer Verlag, 2011; 46

21 Schuetze SM. Utility of positron emission tomography in sarcomas. Curr Opin Oncol 2006; 18: 369-373

22 McCarville MB, Christie R, Daw NC et al. PET/CT in the evaluation of childhood sarcomas. Am J Roentgenol 2005; 184: 1293-1304

23 Schuetze SM. Imaging and response in soft tissue sarcomas. Hematol Oncol Clin North Am 2005; 19: 471-487

24 Saint Aubain Somerhausen $N$ de, Fletcher CD. Soft tissue sarcomas: An update. Eur J Surg Oncol 1999; 25: 215-220

25 Abraham JA, Baldini EH, Butrynski JE. Management of adult soft-tissue sarcoma of the extremities and trunk. Expert Rev Anticancer Ther 2010; 10: 233-248 
26 Tunn PU, Kettelhack C, Durr HR. Standardized approach to the treatment of adult soft tissue sarcoma of the extremities. Recent Results Cancer Res 2009; 179: 211-228

27 Kilpatrick SE, Cappellari JO, Bos GD et al. Is fine needle aspiration biopsy a practical alternative to open biopsy for the primary diagnosis of sarcoma? Experience of 140 patients. Am J Clin Pathol 2001; 115: 59-68

28 Klein T, Hünerbein M. Biopsieverfahren. In: Schlag PM, Hartmann JT, Budach V. Weichgewebetumoren - Interdisziplinäres Management. Berlin, Heidelberg: Springer Verlag, 2011; 68

29 Lehnhardt M, Daigeler A, Homann HH et al. Die Bedeutung von Referenzzentren in Diagnose und Therapie von Weichgewebssarkomen der Extremitäten - Auswertung von 603 Fällen. Chir 2009; 80: 341-347

30 Gronchi A, Lo Vullo S, Colombo C et al. Extremity soft tissue sarcoma in a series of patients treated at a single institution: local control directly impacts survival. Am Surg 2010; 251: 506-511

31 Papagelopoulos PJ, Mavrogenis AF, Mastorakos DP et al. Current concepts for management of soft tissue sarcomas of the extremities. J Surg Orthop Av 2008; 17: 204-215

32 Lehnhardt M, Kuhnen C, Drücke D et al. Liposarkome der Extremitäten Aktuelle Aspekte zur chirurgischen Therapie - Eine Analyse von 167 Patienten. Chirurg 2004; 75: 1182-1190

33 Steinau HU, Steinsträsser L, Langer $S$ et al. Resektionsgrenzen bei Weichgewebssarkomen der Extremitäten. Pathologe 2011; 32: 57-64

34 Dickinson IC, Whitwell DJ, Battistuta $D$ et al. Surgical margin and its influence on survival in soft tissue sarcoma. ANZ J Surg 2006; 76: 104-109

35 Rimner A, Brennan MF, Zhang $Z$ et al. Influence of compartmental involvement on the patterns of morbidity in soft tissue sarcoma of the thigh. Cancer 2009; 115: 149-157

36 Turcotte RE, Ferrone $M$, Isler $M H$ et al. Outcomes in patients with popliteal sarcomas. Can J Surg 2009; 52: 51-55

37 Enneking WF, Spanier SS, Goodman MA. Current concepts review. The surgical staging of musculoskeletal sarcoma. J Bone Jt Surg Am 1980; 62: 1027-1030

38 Mendenhall WM, Indelicato DJ, Scarborough MT et al. The management of adult soft tissue sarcomas. Am J Clin Oncol 2009; 32: 436-442

39 Melcher I, Schaser KD. Weichgewebetumoren der Extremitäten - Operative Verfahren. In: Schlag PM, Hartmann JT, Budach V. Weichgewebetumoren - Interdisziplinäres Management. Berlin, Heidelberg: Springer Verlag, 2011; 76

40 Tanabe KK, Pollock RE, Ellis LM et al. Influence of surgical margins on outcome in patients with preoperatively irradiated extremity soft tissue sarcomas. Cancer 1994; 73: 1652-1659
41 Tunn PU, Andreou D, Illing $H$ et al. Sentinel node biopsy in synovial sarcoma. Eur J Surg Oncol 2008; 34: 704-707

42 Maduekwe UN, Hornicek FJ, Springfield DS et al. Role of sentinel lymph node biopsy in the staging of synovial, epitheloid, and clear cell sarcomas. Ann Surg Oncol 2009; 16: 1356-1363

43 Daigeler A, Lehnhardt $M$, Hauser $J$ et al. Weichgewebetumoren der Extremitäten - Rekonstruktionsverfahren. In: Schlag PM, Hartmann JT, Budach V. Weichgewebetumoren - Interdisziplinäres Management. Berlin, Heidelberg: Springer Verlag, 2011; 98

44 Capanna $R$, Campanacci DA, Belot $N$ et al. A new reconstructive technique for intercalary defects of long bones: the association of massive allograft with vascularized fibular autograft. Long-term results and comparison with alternative techniques. Orthop Clin North Am 2007; 38: 51-60

45 Chang DW, Weber $K L$. Use of a vascularized fibula bone flap and intercalary allograft for diaphyseal reconstruction after resection of primary extremity bone sarcomas. Plast Reconstr Surg 2005; 116 : 1918-1925

46 Germann $G$, Waag $K L$, Selle B et al. Extremity salvage with a free musculocutaneous latissimus dorsi flap and free tendon transfer after resection of a large congenital fibro sarcoma in a 15-week-old infant. Microsurgery 2006; 26: 429-431

47 Daigeler A, Lehnhardt M, Khadra A et al. Proximal major limb amputations - a retrospective analysis of 45 oncological cases. World J Surg Oncol 2009; 7: 15

48 Gustafson P, Dreinhofer KE, Rydholm A. Soft tissue sarcoma should be treated at a tumor center: A comparison of quality of surgery in 375 patients. Acta Orthop Scand 1994; 65: 47-50

49 Reece GP, Schusterman MA, Pollock RE et al. Immediate versus delayed free-tissue transfer salvage of the lower extremity in soft tissue sarcoma patients. Ann Surg Oncol 1994; 1: 11-17

50 Zahlten-Hinguranage A, Bernd L, Ewerbeck $V$ et al. Equal quality of life after limb-sparing or ablative surgery for lower extremity sarcomas. Br J Cancer 2004; 91: 1012-1014

51 Steinau HU, Steinsträsser L, Hauser J et al. Bösartige Weichgewebegeschwülste - Resektion und plastische Wiederherstellung. Chirurg 2012; 83: 673-684

52 Barner-Rasmussen I, Popov P, Bohling $T$ et al. Microvascular reconstruction after resection of soft tissue sarcoma of the leg. Br J Surg 2009; 96: 482-489 